Review Article

\title{
Risk Factors Associated with Irreversible Airway Obstruction in Asthma: A Systematic Review and Meta-Analysis
}

\author{
Lanlan Zhang, ${ }^{1}$ Lixiu He, ${ }^{1}$ Jin Gong, ${ }^{2}$ and Chuntao Liu ${ }^{1}$ \\ ${ }^{1}$ Department of Respiration Medicine, West China Hospital, Sichuan University, Chengdu 610041, China \\ ${ }^{2}$ Hanlin Tongjin Clinic, Chengdu 610071, China \\ Correspondence should be addressed to Chuntao Liu; zll280831@163.com
}

Received 13 November 2015; Revised 3 February 2016; Accepted 11 February 2016

Academic Editor: Cyro A. de Brito

Copyright (c) 2016 Lanlan Zhang et al. This is an open access article distributed under the Creative Commons Attribution License, which permits unrestricted use, distribution, and reproduction in any medium, provided the original work is properly cited.

\begin{abstract}
Irreversible airway obstruction (IAO) is a subtype of asthma and relates to poorer prognosis in some asthma patients. However, the prevalence and risk factors for IAO are unknown. A systematic review regarding controlled clinical studies (cohort, case-control studies) on IAO asthma in adult and/or children affected by asthma/early wheeze was performed. Eighteen papers were identified in this study. It was reported that the incidence of IAO at random effects or fixed effects in severe asthma and nonsevere asthma was 0.54 (95\% CI: $0.45-0.62)$ and 0.16 (95\% CI: 0.12-0.20), respectively. In IAO asthma, the pooled odds ratio (OR) related to smoking exposure was 2.22 (95\% CI: 1.82-2.73), the OR for male, smoking, and fractional exhaled nitric oxide (FENO) was 2.22 (95\% CI: 1.82-2.7), 1.79 (95\% CI: 1.46-2.19), and 2.16 (95\% CI: 1.05-4.43), respectively, suggesting these factors increase the risk of IAO. However, a decreased OR in IAO asthma was observed due to rhinitis (OR $=0.31,95 \% \mathrm{CI}: 0.24-0.40)$, atopy $(\mathrm{OR}=0.584$, 95\% CI: $0.466-0.732)$, and atopic dermatitis ( $\mathrm{OR}=0.60,95 \% \mathrm{CI}: 0.42-0.85)$, indicating these factors are associated with reduced risk of IAO. IAO in asthma is associated with gender, smoking, FENO, rhinitis, atopy, and atopic dermatitis.
\end{abstract}

\section{Introduction}

Asthma is a multifactorial, heterogeneous, and chronic inflammatory disease, which is characterized with the symptoms of cough, shortness of breath, wheeze, and chest tightness. The majority of patients with reversible airflow limitation asthma can be easily controlled by regular medication. However, in a small subset of asthmatic patients, even aggressive treatment fails to control the disease. The prevalence of these patients with so-called irreversible airway obstruction (IAO) asthma is not known.

Backman et al. first reported the patients with IAO asthma [1]. Lange et al. [2] found that asthmatic patients had greater declines in forced expiratory volume in 1 second (FEV1) over time compared to those who did not have asthma. Ulrik and Backer [3] found that IAO adults patients usually are associated with moderate to severe asthma. Some risk factors for IAO development have been defined; IAO asthma is associated with more severe disease [4-6] and is a predictor of overall mortality in asthmatic patients [7]. The reduction of risk factors for IAO may be the key strategy to decrease the morbidity in severe asthmatic patients with IAO. Vonk et al. [8] found that $41 \%$ of asthmatic patients did not have airway obstruction and $16 \%$ of asthmatic patients had IAO. However, studies about the prevalence and risk factors for IAO in asthmatic patients are limited and often contradictory. Martin et al. demonstrated that males have higher possibility than females to develop into asthma patients [9]. Ten Brinke et al. [6] found that the onset of asthma in adults increased AHR and sputum eosinophilia, which increased the risk factor for IAO asthma. Apostol et al. [10] found that smoking is a risk factor for IAO asthma. Aspirin sensitivity has been identified as a risk factor for IAO asthma [11].

Our meta-analysis was designed to assess the prevalence and risk factors of IAO in a well-defined group of patients with asthma/nonsevere asthma and to examine the clinical characteristics. In this study, we analyzed age, gender, disease duration, rhinitis, atopic dermatitis, aspirin sensitivity, smoking history and atopic status, and some clinical characteristics including markers of airway inflammation, fractional exhaled nitric oxide (FENO), and percentage of eosinophils and neutrophils in induced sputum. 


\section{Methods}

According to the rules of the meta-analysis of Observational Studies in Epidemiology group, this meta-analysis was conducted [12]. The literature related to the terms "airways obstruction" OR "irreversible airway obstruction" OR "fixed airflow obstruction" OR "airway remodeling” AND "asthma” was collected from Ovid Medline, Pubmed, and ISI database by two independent reviewers (Z. LL. and G. J., last update in March, 2013). English was included in this meta-analysis. References were also manually searched to identify additional published or unpublished data and were evaluated by experts.

Studies of our meta-analysis included the following inclusion criteria: (1) primary outcome of incidence or risk factors of IAO asthma; (2) follow-up of at least $70 \%$ of patients; (3) all studies that used self-reported, physician-diagnosed asthma or new symptoms and/or medication use compatible with asthma as the criteria for incident asthma diagnosis. We excluded the studies that used single asthma symptom as an outcome and did not establish asthma diagnosis. Moreover, we did not consider those studies that did not report the odds risk. Study data sources were examined to ensure that every included dataset was unique.

Stata 12.0 (Stata Corporation, College Station, TX) [13] was used to calculate ORs using inverse-variance weighted, random-effects meta-analysis [14, 15]. Randomeffects methodology was chosen to analyze within-study and between-study variations. Heterogeneity of data was evaluated using the Q-statistic [15]. We weighted the study-specific adjusted log odd risks for cohort studies and the studyspecific adjusted log odds ratios for case-control studies by inversing their variance to compute a pooled odds risk and 95\% confidence intervals (CIs) on a forest plot [16], and publication bias was evaluated [16, 17]. Using data from studies that provided mean repeat length of specific case groups, we calculated standardized mean difference (SMD) to compare IAO cases with reversible airway obstruction (RAO) controls. The SMD and 95\% CI were calculated in each group of cases. A plan was established prior to performing sensitivity analyses for identified issues relative to study quality, if necessary, rather than applying weights to studies in the meta-analysis based simply on quality scoring criteria [12].

$\chi^{2}$-based $Q$ statistic evaluated heterogeneity and considered the statistical significance at $I^{2}$-value less than $50 \%$. When the $I^{2}$-value is less than $50 \%$, fixed-effect model was used; Otherwise, a random-effects model was applied. The significance of the pooled OR was determined by the $Z$-test, and $P$ less than 0.05 was considered statistically significant. Publication bias was analyzed by several methods: (i) funnel plots; (ii) Egger's test that was also used to statistically assess publication bias. All statistical tests were performed by using the Revman 5.0 software and Stata 12.0.

\section{Results}

According to our search terms, 343 references were retrieved, but only 32 articles met our inclusion criteria. Among them, some references that only presented cross-sectional data and failed to present any measurement of the odds risk for IAO were further excluded [2, 9, 13, 18-32]. Only 18 studies were included in this study [3-6, 8, 11,30-40], which included one study from persistent wheezing but not from asthma [41]. All 18 cohort studies were published between 1995 and 2013 (Table 1).

\section{Incidence of IAO Asthma}

Twelve articles with the data of risk factors for IAO were retrieved [3-6, 8, 11, 33, 34, 36, 38, 39, 41]. Of the total 13,498 patients, $16 \%$ of patients were diagnosed with IAO. Four articles were involved in severe asthma [4-6, 34], in which $54 \%$ of patients were analyzed by asthma subtypes and IAO asthma using the random-effects model. The incidence of IAO asthma in non-severe asthma patients was $16 \%$ with the random-effects model (Figure 1) or $8 \%$ with the fixed-effect model.

\section{Risk Factors}

5.1. Male. Ten studies $[4,6,8,31,32,34-37,42]$ were carried out in a case-control study, the pooled odds risk for male by fixed-effect model was 1.622 (95\% CI: 1.358-1.937), and there was a considerable heterogeneity among studies $\left(I^{2}=11.6 \%\right)$ (Figure 2).

5.2. Smoking. Eight articles were retrieved based on smoking for IAO asthma patients in a cohort study [4-6, 33-35, 37, 39]. The amount of heterogeneity was decreased $\left(I^{2}=38.2 \%\right)$ when we set our analysis to case-control studies; the patients exposed to smoking showed the pooled OR of 1.79 (95\% CI: 1.46-2.19). Moreover, we did not find any evidence of publication bias ( $P$ value of Egger's symmetry test: 0.606$)$ (Figure 3).

5.3. Atopy. Eight studies related to atopy were included in this study $[4,5,8,31,32,34-36]$; the overall OR was $0.584(95 \%$ CI: $0.466-0.732)$ by fixed-effect model. There was a small heterogeneity $I^{2}=22.2 \%$ (Figure 4 ).

5.4. Rhinitis. Three studies were associated with rhinitis [5, $35,41]$. Rhinitis showed a protective effect on IAO asthma $(\mathrm{OR}=0.31,95 \% \mathrm{CI}: 0.24-0.40)$ by a fixed-effect model. There was no heterogeneity $\left(I^{2}=0 \%\right)$ between IAO asthma studies and RAO asthma studies (Figure 5).

5.5. Aspirin Sensitivity. Two studies relative to aspirin sensitivity were included $[5,11]$. The fixed-effect model generated the OR of 2.053 (95\% CI: 1.417-2.689), but there was significant heterogeneity by random-effects model $\left(I^{2}=94.4 \%\right)$. Although the result showed the heterogeneity, both of them showed that aspirin sensitivity was an important risk factor for IAO.

5.6. FENO. Two studies related to FENO were included [6, 34]. There was no heterogeneity; the result from fixed-effect 


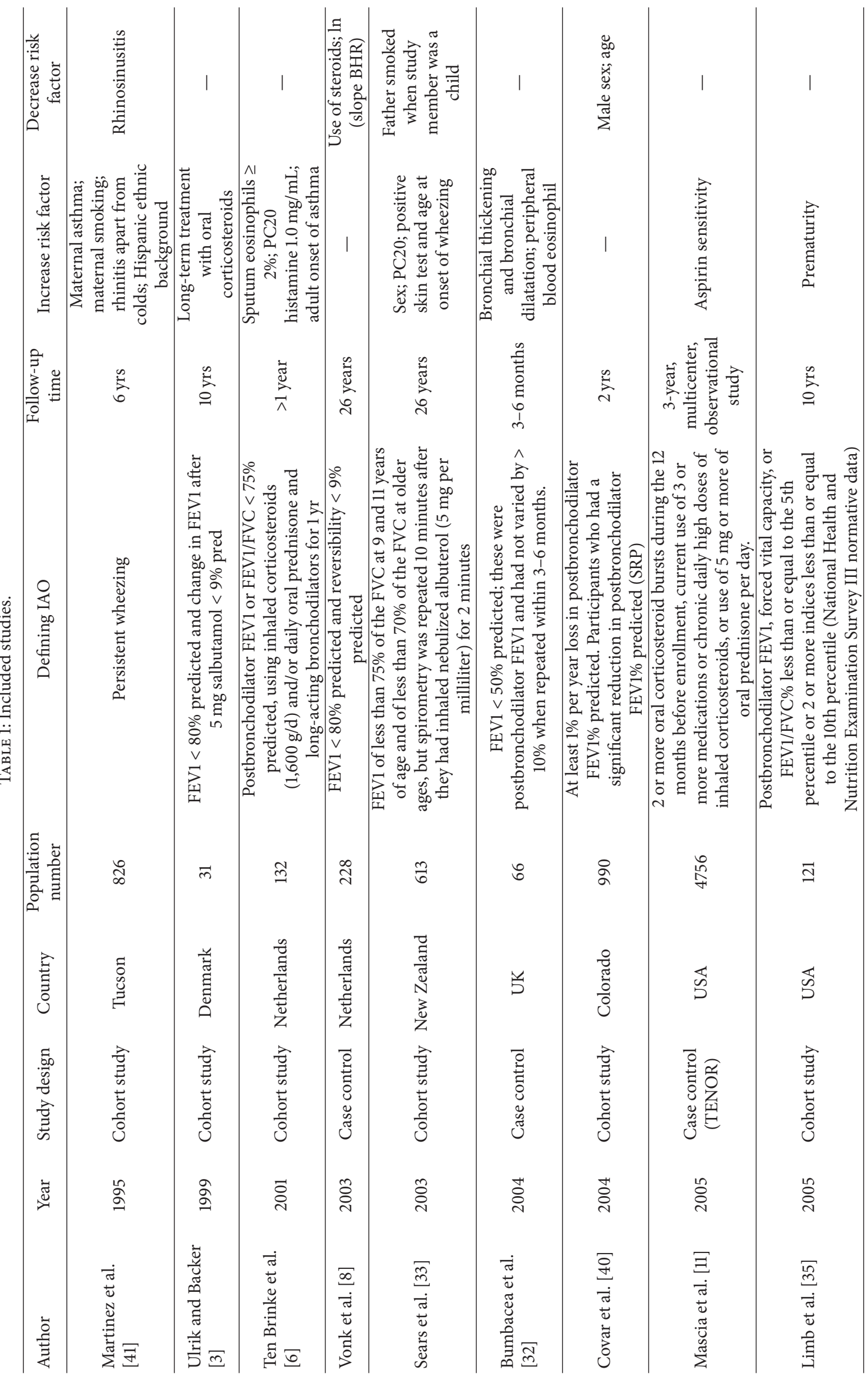




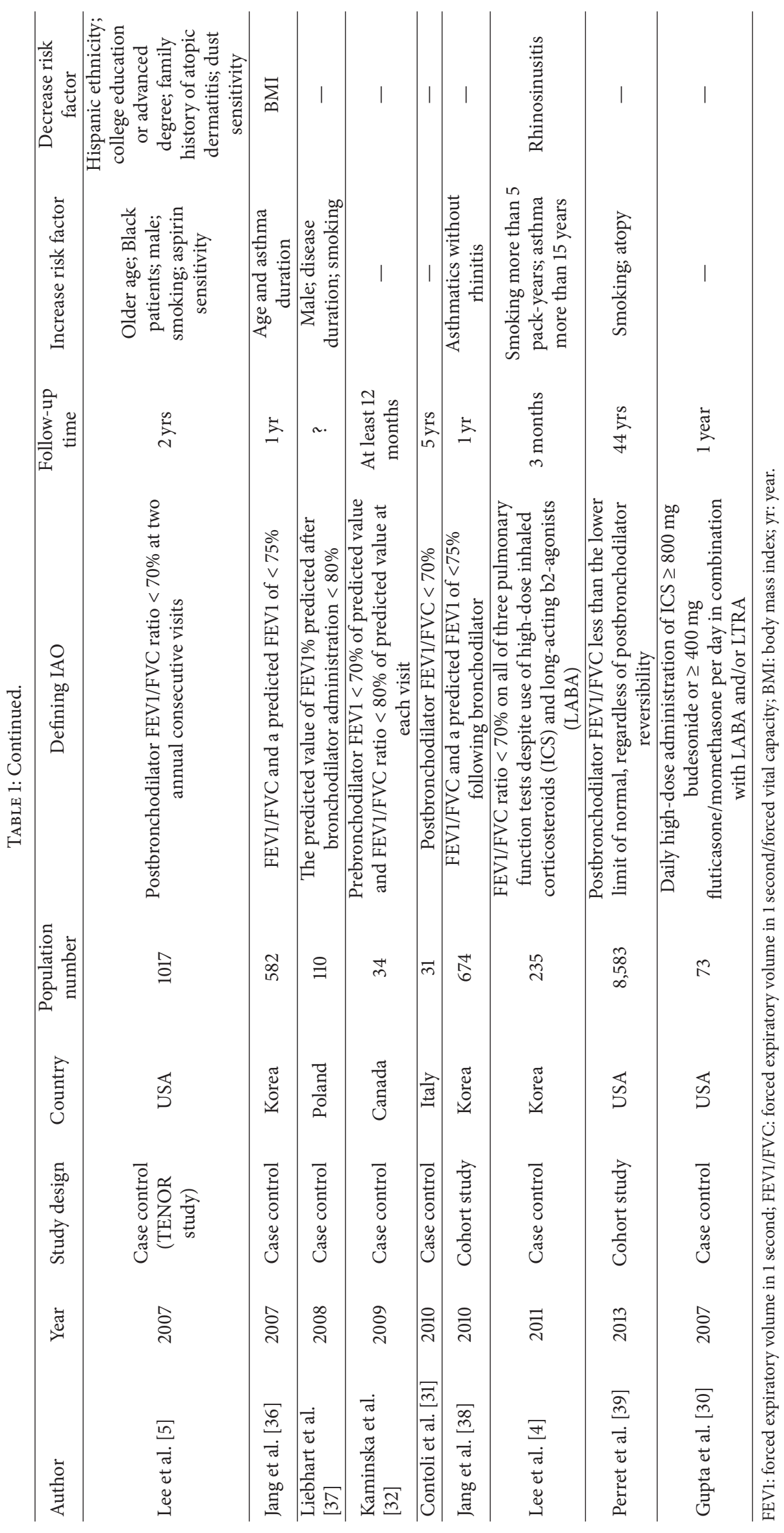




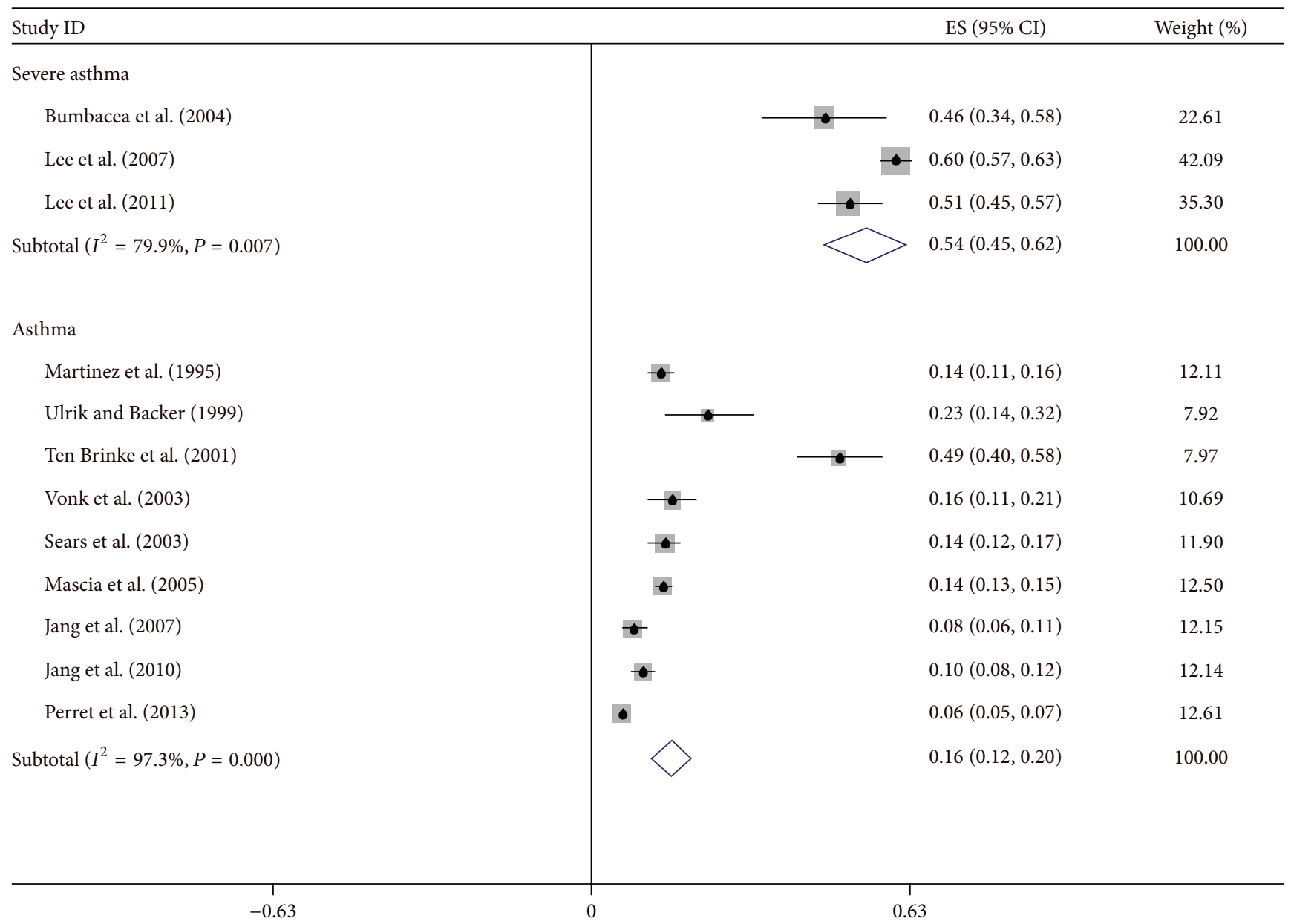

FIGURE 1: The summary for the odds of incident IAO asthma by a random-effects model. Numbers indicate reference number.

model was similar to that from random-effects model. These two studies yielded a protective pooled odds risk of 2.16 with $95 \%$ CI: 1.05 to 4.43 .

5.7. Atopic Dermatitis. Two studies focused on atopic dermatitis [5, 34]. There was no heterogeneity, so fixed-effect pooled model was used. These two studies yielded a protective pooled odds risk of 0.60 with $95 \%$ CI: 0.42 to 0.85 .

5.8. Others. The results showed that age at onset, asthma duration, sputum eosinophils, blood eosinophils, and atopy were not important discriminators for airway remodeling in cohort studies.

Five studies found that OR age at onset is another risk factor in IAO asthma patients with OR of 1.04 (95\% CI: 1.01$1.06)$ by the fixed-effect model $[4,5,8,36,40]$, but significant heterogeneity $\left(I^{2}=92.4 \%\right)$ was observed among studies and the random-effects model showed different result $(\mathrm{OR}=$ 1.02 , 95\% CI: $0.88-1.19)$. The amount of heterogeneity did not decrease when the sensitivity analysis was performed (data not shown). We also did not find any evidence of publication bias ( $P$ value of Egger's symmetry test: 1.00).
Compared with RAO asthma patients, we found longer disease duration $(\mathrm{SMD}=2.84,95 \% \mathrm{CI}: 1.22-4.45)$, lower sputum eosinophils (SMD $=-0.99,95 \% \mathrm{CI}: 1.20-0.79)$, and lower body mass index (BMI) $(\mathrm{SMD}=-1.155,95 \% \mathrm{CI}$ : $-1.348-0.962$ ) in IAO asthma patients (Table 2).

\section{Discussion}

To our knowledge, this is the first meta-analysis study for the prevalence and the risk factors of IAO. Our findings support the high prevalence of IAO in severe asthma. IAO is highly associated with smoking and male asthma patients have twofold greater risk of IAO. Furthermore, the asthma patients with higher FENO and aspirin sensitivity also have two-fold greater risk of IAO. However, atopic dermatitis and rhinitis and atopy were significantly related to the decreasing IAO in severe asthma.

The concept of IAO implies that some changes in the structure and function of the airway occur and reduce expiratory airflow, and these changes cannot be restored to the prior state by endogenous mechanism or any treatment. Some indirect evidence demonstrated that progressive airflow obstruction may cause IAO because severe or 
TABLE 2: Pooled odds risks (OR) and 95\% confidence intervals (CIs) of irreversible airway obstruction asthma and risk factor in cohort or case control study.

\begin{tabular}{|c|c|c|c|c|}
\hline Type & Number of studies & $\begin{array}{l}\text { OR }(95 \% \text { CI }) \\
\text { Fixed effects }\end{array}$ & $\begin{array}{c}\text { OR (95\% CI) } \\
\text { Random effects }\end{array}$ & $I^{2}$ \\
\hline$\overline{\text { Age }}$ & 5 (cohort) & $1.037(1.012,1.062)$ & $1.023(0.878,1.192)$ & $92.4 \%(\mathrm{R})$ \\
\hline Male & 10 (case control) & $2.222(1.821,2.731)$ & & $53.3 \%(\mathrm{~F})$ \\
\hline Age at onset & 4 (cohort) & $0.971(0.937,1.006)$ & $1.166(0.646,2.103)$ & $47.2 \%(\mathrm{R})$ \\
\hline Disease duration & 5 (cohort) & $1.062(1.038,1.086)$ & $1.158(1.026,1.308)$ & $91.6 \%(\mathrm{R})$ \\
\hline Rhinitis & 3 (case control) & $0.31(0.24,0.40)$ & & $0.0 \%(\mathrm{~F})$ \\
\hline Atopic dermatitis & 2 (cohort) & $0.599(0.424,0.848)$ & & $2.1 \%(\mathrm{~F})$ \\
\hline Aspirin sensitivity & 2 (cohort) & $2.053(1.417,2.689)$ & $1.826(0.263,12.693)$ & $92.4 \%(\mathrm{R})$ \\
\hline Smoking & 9 (case control) & $1.79(1.46,2.19)$ & - & $38.2 \%(F)$ \\
\hline Sputum eosinophils & 2 (cohort) & $0.979(0.937,1.022)$ & $2.514(0.334,18.893)$ & $91.6 \%(\mathrm{R})$ \\
\hline Blood eosinophils & 2 (cohort) & $2.031(0.958,4.304)$ & & $32.4 \%(\mathrm{~F})$ \\
\hline FENO & 2 (cohort) & $2.156(1.050,4.425)$ & & $0.0 \%(\mathrm{~F})$ \\
\hline Atopy & 8 (case control) & $0.584(0.466,0.732)$ & - & $22.2(\mathrm{~F})$ \\
\hline
\end{tabular}

R: randomised effects; F: fixed effects.

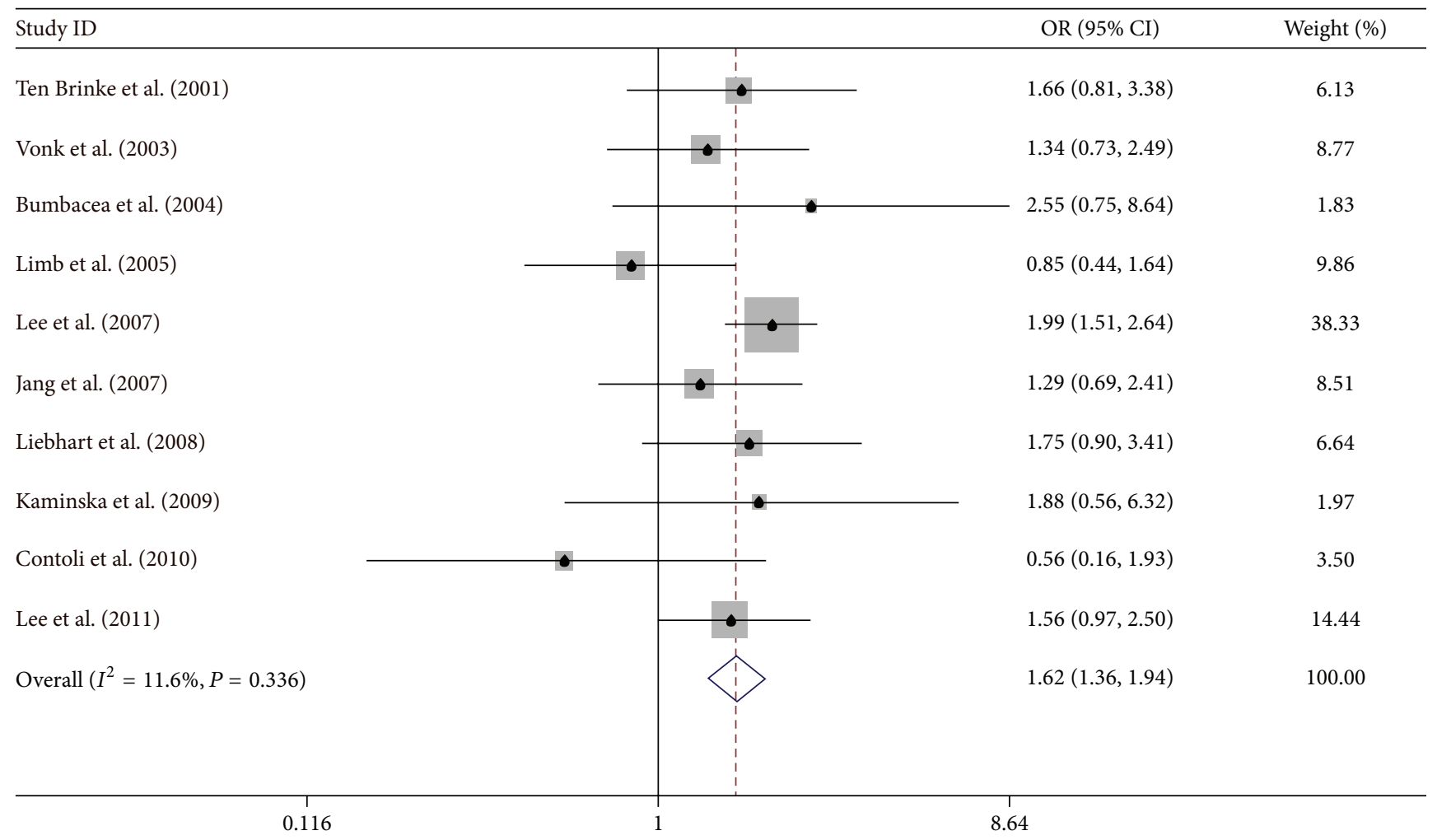

FIGURE 2: Meta-analysis for the associations between IAO asthma risk and male gender.

progressive airflow obstruction occurs in some IAO patients in spite of ongoing anti-inflammatory (glucocorticoid) or bronchodilator treatment. The studies on the pathogenesis of airway remodeling in asthma [43], such as airway wall thickness, allergic airway inflammation, epithelial-driven models of airway remodeling, bronchial neovascularization, and physiological consequences of airway remodeling, support explaining the reasons of IAO.
Some studies have explored the risk factors for the development of IAO in asthma. The reduction in lung function in children and adults may contribute to the development of IAO. Cigarette smoking is related to the development of IAO asthma and has been associated with a rapid decline in lung function in asthma $[2,44]$. It might be related to smoking-induced changes in airway inflammation, with a predominance of neutrophils instead of eosinophils [45]. 


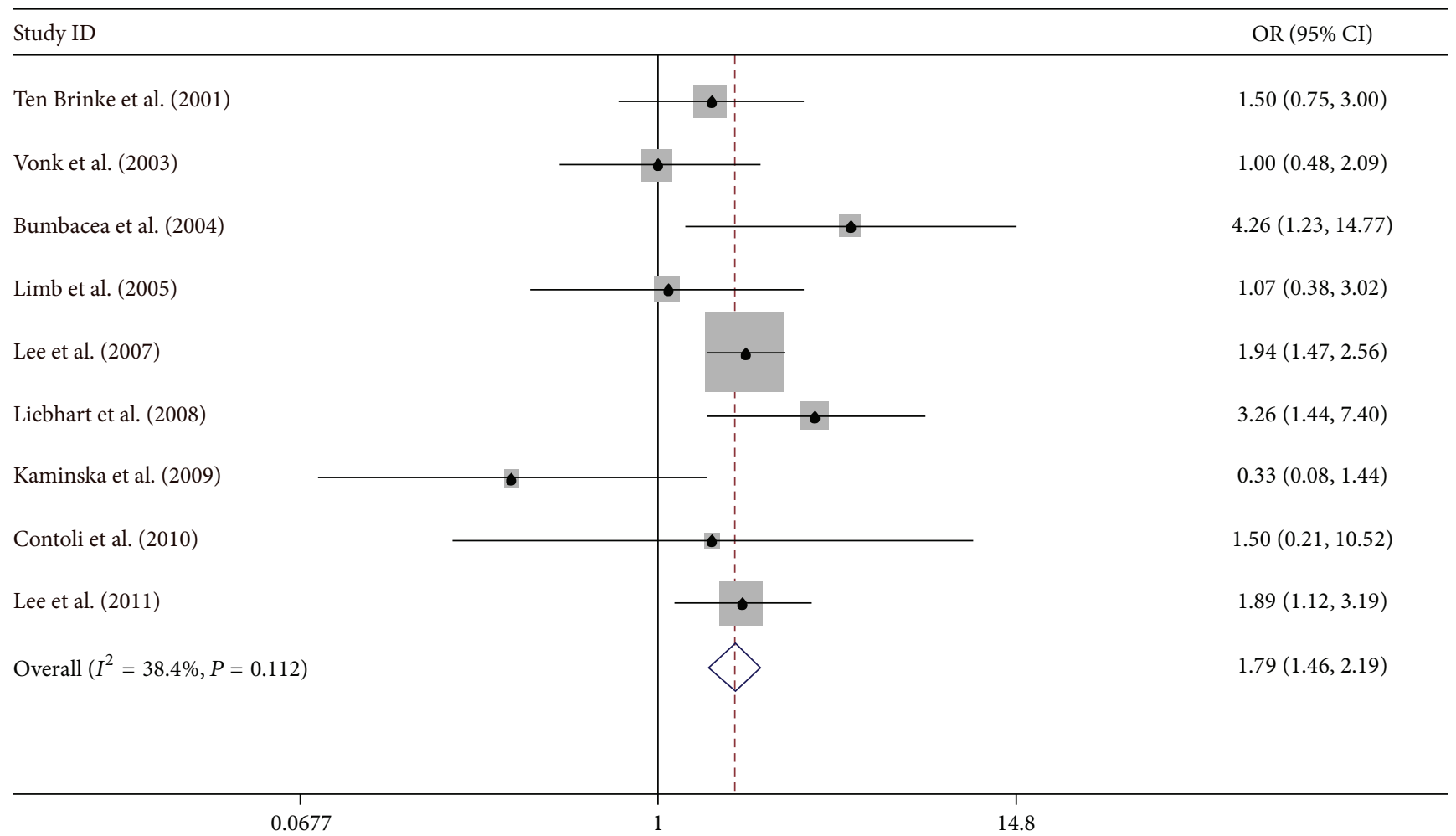

FIGURE 3: Meta-analysis for the associations between IAO asthma risk and smoking.

\begin{tabular}{|c|c|}
\hline Study ID & OR $(95 \% \mathrm{CI})$ \\
\hline Vonk et al. (2003) & $0.54(0.23,1.27)$ \\
\hline Bumbacea et al. (2004) & $0.99(0.37,2.61)$ \\
\hline Limb et al. (2005) & $0.97(0.41,2.32)$ \\
\hline Lee et al. (2007) & $0.57(0.40,0.80)$ \\
\hline Jang et al. (2007) & $0.35(0.19,0.66)$ \\
\hline Kaminska et al. (2009) & $1.67(0.33,8.46)$ \\
\hline Contoli et al. (2010) & $3.75(0.34,40.81)$ \\
\hline Lee et al. (2011) & $0.52(0.31,0.89)$ \\
\hline Overall $\left(I^{2}=22.2 \%, P=0.253\right)$ & $0.58(0.47,0.73)$ \\
\hline
\end{tabular}

FIgURE 4: Meta-analysis for the associations between IAO asthma risk and rhinitis.

Jiang et al. [46] suggested that atopy in itself is not related to asthma. However, asthma and atopy usually occur in parallel. Since atopic asthma is usually mild and occurs early, it is more commonly accompanied by other allergic diseases such as allergic rhinitis [47]. A reduced prevalence of rhinosinusitis in patients with more severe asthma may be due to the spontaneous regression of rhinosinusitis during the course of asthma [48]. Mascia et al. [11] showed that adults 


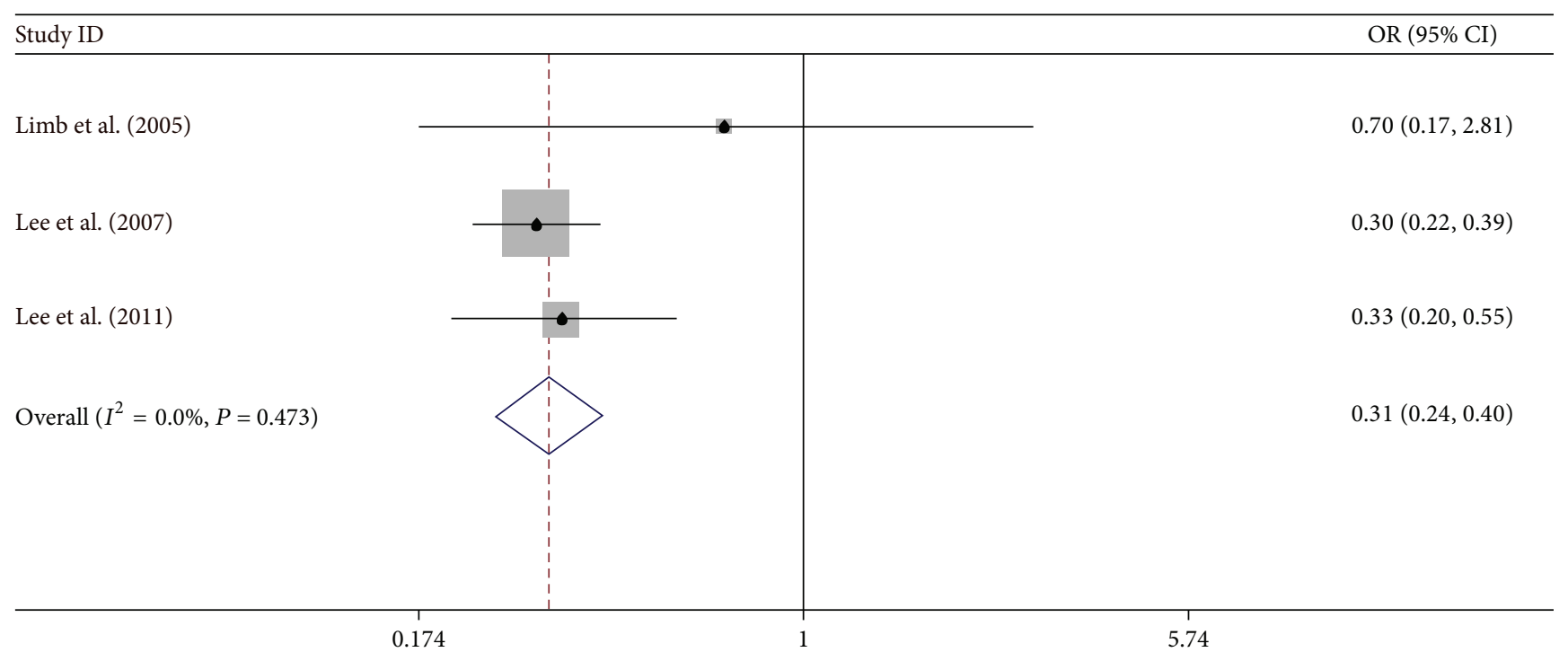

FIgURE 5: Meta-analysis for the associations between IAO asthma risk and atopy.

with aspirin-sensitive asthma have more severe disease and airway obstruction than aspirin-insensitive asthma patients. It may be possible that aspirin-sensitive asthma patients have longer disease duration. Chronic inflammation of the airways may also contribute to IAO development, so it may explain why FENO is the major factor of IAO.

Two major mechanisms for airway remodeling including reticular basement membrane (RBM) and subepithelial basement membrane thickening have been reported $[49,50]$. Another common finding in remodeled airways is the presence of increased airway smooth muscle mass [51]. Findings of increased RBM thickness and airway smooth muscle area on biopsies of severe asthma patients have intrigued the interest in the use of noninvasive imaging techniques, such as computed tomography (CT) [52], to assess the changes in the bronchial wall thickness and structure. The third dogma is that the structure changes that cause the functional changes in the airway wall are primarily responsible for IAO. In the future, we still use the risk factors to explore and identify the mechanisms of remodeling.

The limitations of this meta-analysis should also be addressed. First, some studies are excluded due to the absence of original dada, which may lead to selection bias. Thus, these studies are usually excluded. Second, all eligible studies are published in English from selected databases. It is possible that some relevant studies published in other languages are missed. Third, most studies are from Asians and Caucasians; thus our study may be applicable to non-Africans only. Fourth, asthma is a heterogeneous disease, and the therapy may affect risk factors. Fifth, we should also analyze the possibility of publication bias. Publication bias can result in the disappearance of some studies with negative results.

In conclusion, this study is the first to report risk factors that may be related to IAO in asthma patients. We found that smoking, male gender, FENO, and the absence of rhinitis, atopy, and atopic dermatitis were more likely to lead to persistent IAO. In addition, our findings further demonstrated that smoking is an important cause of irreversible airway damage. Further we need more studies to clarify the underlying risk factors of the development of IAO in subjects with asthma. The search for known and novel therapies that can directly target individual components of the remodeling process and hopefully lead to an improvement in the treatment of airflow limitation and the prognosis in severe asthma will be made.

\section{Competing Interests}

The authors have declared that no competing interests exist.

\section{Authors' Contributions}

Lanlan Zhang and Lixiu He contributed equally to this work.

\section{Acknowledgments}

This paper was supported by National Nature Science Foundation of China (NSFC): 0040205401604.

\section{References}

[1] K. S. Backman, P. A. Greenberger, and R. Patterson, "Airways obstruction in patients with long-term asthma consistent with 'irreversible asthma,' Chest, vol. 112, no. 5, pp. 1234-1240, 1997.

[2] P. Lange, J. Parner, J. Vestbo, P. Schnohr, and G. Jensen, "A 15-year follow-up study of ventilatory function in adults with asthma," The New England Journal of Medicine, vol. 339, no. 17, pp. 1194-200, 1998.

[3] C. S. Ulrik and V. Backer, "Nonreversible airflow obstruction in life-long nonsmokers with moderate to severe asthma," European Respiratory Journal, vol. 14, no. 4, pp. 892-896, 1999.

[4] T. Lee, Y. S. Lee, Y.-J. Bae et al., "Smoking, longer disease duration and absence of rhinosinusitis are related to fixed airway obstruction in Koreans with severe asthma: findings 
from the COREA study," Respiratory Research, vol. 12, article 1, 2011.

[5] J. H. Lee, T. Haselkorn, L. Borish, L. Rasouliyan, B. E. Chipps, and S. E. Wenzel, "Risk factors associated with persistent airflow limitation in severe or difficult-to-treat asthma," Chest, vol. 132, no. 6, pp. 1882-1889, 2007.

[6] A. Ten Brinke, A. H. Zwinderman, P. J. Sterk, K. F. Rabe, and E. H. Bel, "Factors associated with persistent airflow limitation in severe asthma," American Journal of Respiratory and Critical Care Medicine, vol. 164, no. 5, pp. 744-748, 2001.

[7] E. F. Hansen, K. Phanareth, L. C. Laursen, A. Kok-Jensen, and A. Dirksen, "Reversible and irreversible airflow obstruction as predictor of overall mortality in asthma and chronic obstructive pulmonary disease," American Journal of Respiratory and Critical Care Medicine, vol. 159, no. 4 I, pp. 1267-1271, 1999.

[8] J. M. Vonk, H. Jongepier, C. I. M. Panhuysen, J. P. Schouten, E. R. Bleecker, and D. S. Postma, "Risk factors associated with the presence of irreversible airflow limitation and reduced transfer coefficient in patients with asthma after 26 years of follow up," Thorax, vol. 58, no. 4, pp. 322-327, 2003.

[9] A. J. Martin, L. A. McLennan, L. I. Landau, and P. D. Phelan, "The natural history of childhood asthma to adult life," British Medical Journal, vol. 280, no. 6229, pp. 1397-1400, 1980.

[10] G. G. Apostol, D. R. Jacobs Jr., A. W. Tsai et al., "Early life factors contribute to the decrease in lung function between ages 18 and 40: the coronary artery risk development in young adults study," American Journal of Respiratory and Critical Care Medicine, vol. 166, no. 2, pp. 166-172, 2002.

[11] K. Mascia, T. Haselkorn, Y. M. Deniz, D. P. Miller, E. R. Bleecker, and L. Borish, "Aspirin sensitivity and severity of asthma: evidence for irreversible airway obstruction in patients with severe or difficult-to-treat asthma," The Journal of Allergy and Clinical Immunology, vol. 116, no. 5, pp. 970-975, 2005.

[12] D. F. Stroup, J. A. Berlin, S. C. Morton et al., "Meta-analysis of observational studies in epidemiology," The Journal of the American Medical Association, vol. 283, no. 15, pp. 2008-2012, 2000.

[13] S. R. Kim and Y. K. Rhee, "Overlap between asthma and COPD: where the two diseases converge," Allergy, Asthma \& Immunology Research, vol. 2, no. 4, pp. 209-214, 2010.

[14] R. DerSimonian and N. Laird, "Meta-analysis in clinical trials," Controlled Clinical Trials, vol. 7, no. 3, pp. 177-188, 1986.

[15] W. G. Cochran, "The combination of estimates from different experiments," Biometrics, vol. 10, no. 1, pp. 101-129, 1954.

[16] C. B. Begg and M. Mazumdar, "Operating characteristics of a rank correlation test for publication bias," Biometrics, vol. 50, no. 4, pp. 1088-1101, 1994.

[17] M. Egger, G. D. Smith, M. Schneider, and C. Minder, "Bias in meta-analysis detected by a simple, graphical test," British Medical Journal, vol. 315, no. 7109, pp. 629-634, 1997.

[18] L. M. Fabbri, M. Romagnoli, L. Corbetta et al., "Differences in airway inflammation in patients with fixed airflow obstruction due to asthma or chronic obstructive pulmonary disease," American Journal of Respiratory and Critical Care Medicine, vol. 167, no. 3, pp. 418-424, 2003.

[19] The Childhood Asthma Management Program Research Group, "Long-term effects of budesonide or nedocromil in children with asthma," The New England Journal of Medicine, vol. 343, no. 15, pp. 1054-1063, 2000.

[20] R. C. Strunk, S. T. Weiss, K. P. Yates, J. Tonascia, R. S. Zeiger, and S. J. Szefler, "Mild to moderate asthma affects lung growth in children and adolescents," Journal of Allergy and Clinical Immunology, vol. 118, no. 5, pp. 1040-1047, 2006.

[21] L. Lowe, C. S. Murray, A. Custovic, B. M. Simpson, P. M. Kissen, and A. Woodcock, "Specific airway resistance in 3-year-old children: a prospective cohort study," The Lancet, vol. 359, no. 9321, pp. 1904-1908, 2002.

[22] A. Dijkstra, J. M. Vonk, H. Jongepier et al., "Lung function decline in asthma: association with inhaled corticosteroids, smoking and sex," Thorax, vol. 61, no. 2, pp. 105-110, 2006.

[23] P. D. Phelan, C. F. Robertson, and A. Olinsky, "The Melbourne asthma study: 1964-1999," The Journal of Allergy and Clinical Immunology, vol. 109, no. 2, pp. 189-194, 2002.

[24] W. J. Morgan, D. A. Stern, D. L. Sherrill et al., "Outcome of asthma and wheezing in the first 6 years of life followup through adolescence," American Journal of Respiratory and Critical Care Medicine, vol. 172, no. 10, pp. 1253-1258, 2005.

[25] H. A. Jenkins, R. Cherniack, S. J. Szefler, R. Covar, E. W. Gelfand, and J. D. Spahn, "A comparison of the clinical characteristics of children and adults with severe asthma," Chest, vol. 124, no. 4, pp. 1318-1324, 2003.

[26] E. Horak, A. Lanigan, M. Roberts et al., "Longitudinal study of childhood wheezy bronchitis and asthma: outcome at age 42," British Medical Journal, vol. 326, no. 7386, pp. 422-423, 2003.

[27] P. M. Matricardi, S. Illi, C. Grüber et al., "Wheezing in childhood: incidence, longitudinal patterns and factors predicting persistence," European Respiratory Journal, vol. 32, no. 3, pp. 585-592, 2008.

[28] J. A. Panizza, A. L. James, G. Ryan, N. de Klerk, and K. E. Finucane, "Mortality and airflow obstruction in asthma: a 17year follow-up study," Internal Medicine Journal, vol. 36, no. 12, pp. 773-780, 2006.

[29] J. K. Peat, A. J. Woolcock, and K. Cullen, "Decline of lung function and development of chronic airflow limitation: a longitudinal study of non-smokers and smokers in Busselton, Western Australia," Thorax, vol. 45, no. 1, pp. 32-37, 1990.

[30] S. Gupta, S. Siddiqui, P. Haldar et al., "A qualitative analysis of HRCT scans in difficult asthma," Thorax, vol. 62, p. A7, 2007.

[31] M. Contoli, S. Baraldo, B. Marku et al., "Fixed airflow obstruction due to asthma or chronic obstructive pulmonary disease: 5-year follow-up," Journal of Allergy and Clinical Immunology, vol. 125, no. 4, pp. 830-837, 2010.

[32] M. Kaminska, S. Foley, K. Maghni et al., "Airway remodeling in subjects with severe asthma with or without chronic persistent airflow obstruction," The Journal of Allergy and Clinical Immunology, vol. 124, no. 1, pp. 45.e4-51.e4, 2009.

[33] M. R. Sears, J. M. Greene, A. R. Willan et al., "A longitudinal, population-based, cohort study of childhood asthma followed to adulthood," The New England Journal of Medicine, vol. 349, no. 15, pp. 1414-1422, 2003.

[34] D. Bumbacea, D. Campbell, L. Nguyen et al., "Parameters associated with persistent airflow obstruction in chronic severe asthma," European Respiratory Journal, vol. 24, no. 1, pp. 122 $128,2004$.

[35] S. L. Limb, K. C. Brown, R. A. Wood et al., "Irreversible lung function deficits in young adults with a history of childhood asthma," Journal of Allergy and Clinical Immunology, vol. 116, no. 6, pp. 1213-1219, 2005.

[36] A.-S. Jang, J.-H. Lee, W. P. Sung, J.-S. Park, D.-J. Kim, and C.-S. Park, "Risk factors related to fixed airway obstruction in patients with asthma after antiasthma treatment," Annals of Allergy, Asthma and Immunology, vol. 99, no. 5, pp. 408-412, 2007. 
[37] J. Liebhart, M. Polak, A. Dabrowski et al., "The G/G genotype of transforming growth factor beta 1 (TGF- $\beta 1$ ) single nucleotide $(+915 \mathrm{G} / \mathrm{C})$ polymorphism coincident with other host and environmental factors is associated with irreversible bronchoconstriction in asthmatics," International Journal of Immunogenetics, vol. 35, no. 6, pp. 417-422, 2008.

[38] A.-S. Jang, J.-S. Park, J.-H. Lee et al., "Asthmatics without rhinitis have more fixed airway obstruction than those with concurrent rhinitis," Allergy, Asthma \& Immunology Research, vol. 2, no. 2, pp. 108-113, 2010.

[39] J. L. Perret, S. C. Dharmage, M. C. Matheson et al., "The interplay between the effects of lifetime asthma, smoking, and atopy on fixed airflow obstruction in middle age," American Journal of Respiratory and Critical Care Medicine, vol. 187, no. 1, pp. 42-48, 2013.

[40] R. A. Covar, J. D. Spahn, J. R. Murphy, and S. J. Szefler, "Progression of asthma measured by lung function in the childhood asthma management program," American Journal of Respiratory and Critical Care Medicine, vol. 170, no. 3, pp. 234241, 2004.

[41] F. D. Martinez, A. L. Wright, L. M. Taussig et al., "Asthma and wheezing in the first six years of life," The New England Journal of Medicine, vol. 332, no. 3, pp. 133-138, 1995.

[42] Y. Chen, W. Wei, H. Wu, L.-L. Zhang, and J.-Y. Chen, "Effects of paeoniflorin on the level of antibodies and cAMP produced by splenocytes in rats with adjuvant arthritis," Yao Xue Xue Bao, vol. 42 , no. 11, pp. 1147-1151, 2007.

[43] A. Shifren, C. Witt, C. Christie, and M. Castro, "Mechanisms of remodeling in asthmatic airways," Journal of Allergy, vol. 2012, Article ID 316049, 12 pages, 2012.

[44] A. L. James, L. J. Palmer, E. Kick et al., "Decline in lung function in the Busselton Health Study: the effects of asthma and cigarette smoking," American Journal of Respiratory and Critical Care Medicine, vol. 171, no. 2, pp. 109-114, 2005.

[45] L.-P. Boulet, C. Lemière, F. Archambault, G. Carrier, M. C. Descary, and F. Deschesnes, "Smoking and asthma: clinical and radiologic features, lung function, and airway inflammation," Chest, vol. 129, no. 3, pp. 661-668, 2006.

[46] J. Jiang, K. J. Marienau, L. A. May et al., "Laboratory diagnosis of two scrub typhus outbreaks at Camp Fuji, Japan in 2000 and 2001 by enzyme-linked immunosorbent assay, rapid flow assay, and Western blot assay using outer membrane 56$\mathrm{kD}$ recombinant proteins," The American Journal of Tropical Medicine and Hygiene, vol. 69, no. 1, pp. 60-66, 2003.

[47] B. Sibbald and E. Rink, "Epidemiology of seasonal and perennial rhinitis: clinical presentation and medical history," Thorax, vol. 46, no. 12, pp. 895-901, 1991.

[48] A. Navarro, A. Valero, B. Julia, and S. Quirce, "Coexistence of asthma and allergic rhinitis in adult patients attending allergy clinics: ONEAIR study," Journal of Investigational Allergology and Clinical Immunology, vol. 18, no. 4, pp. 233-238, 2008.

[49] P. K. Jeffery, R. W. Godfrey, E. Ädelroth, F. Nelson, A. Rogers, and S.-A. Johansson, "Effects of treatment on airway inflammation and thickening of basement membrane reticular collagen in asthma: a quantitative light and electron microscopic study," The American Review of Respiratory Disease, vol. 145, no. 4 I, pp. 890-899, 1992.

[50] C. Ward, M. Pais, R. Bish et al., "Airway inflammation, basement membrane thickening and bronchial hyperresponsiveness in asthma," Thorax, vol. 57, no. 4, pp. 309-316, 2002.

[51] C. Pepe, S. Foley, J. Shannon et al., "Differences in airway remodeling between subjects with severe and moderate asthma," The Journal of Allergy and Clinical Immunology, vol. 116 , no. 3, pp. 544-549, 2005.

[52] M. R. Zeidler, E. C. Kleerup, H. Kim, V. Balasubramanian, D. P. Tashkin, and J. Goldin, "Correlations between air trapping on HRCT and asthma symptoms in mild asthmatics," American Journal of Respiratory and Critical Care Medicine, vol. 179, abstract A1268, 2009. 


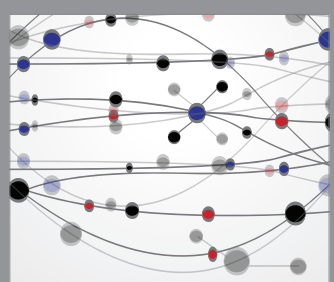

The Scientific World Journal
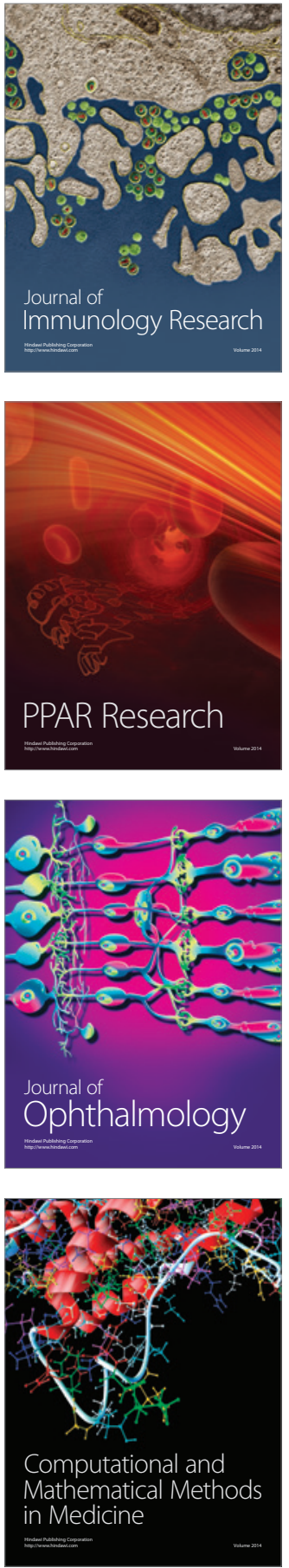

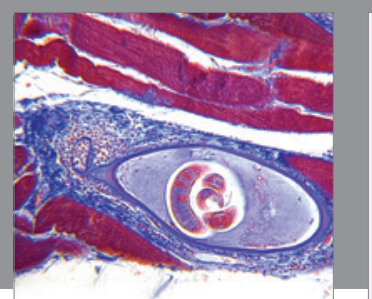

Gastroenterology Research and Practice

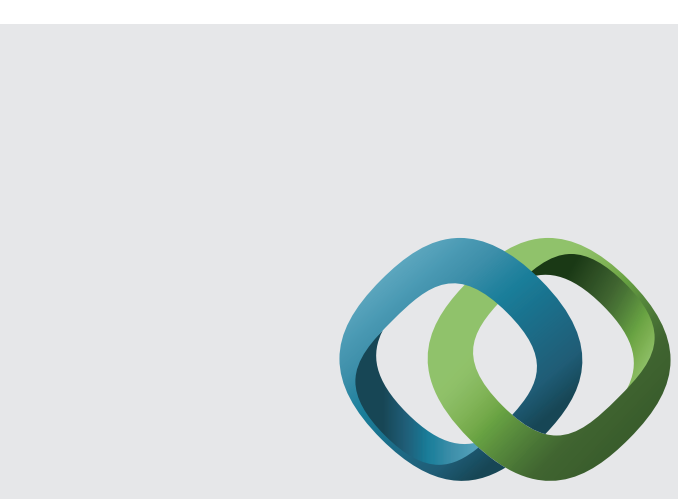

\section{Hindawi}

Submit your manuscripts at

http://www.hindawi.com
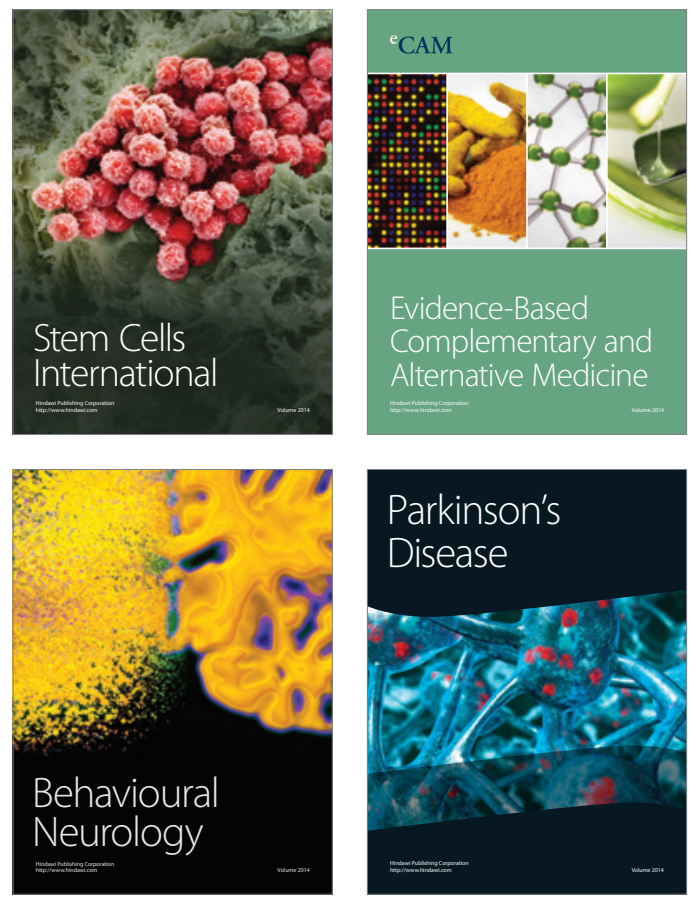
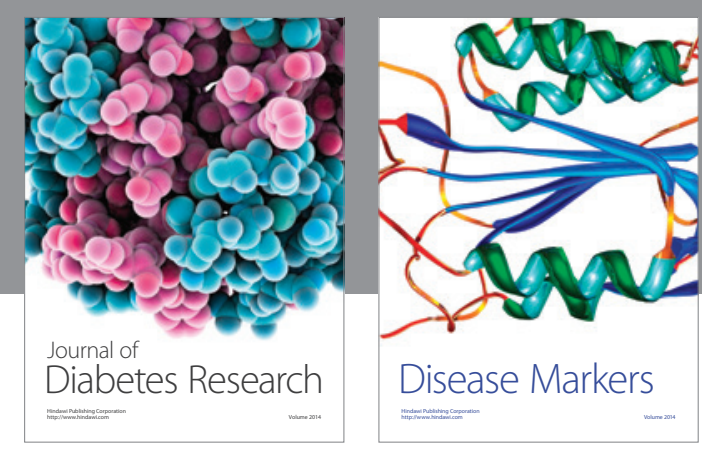

Disease Markers
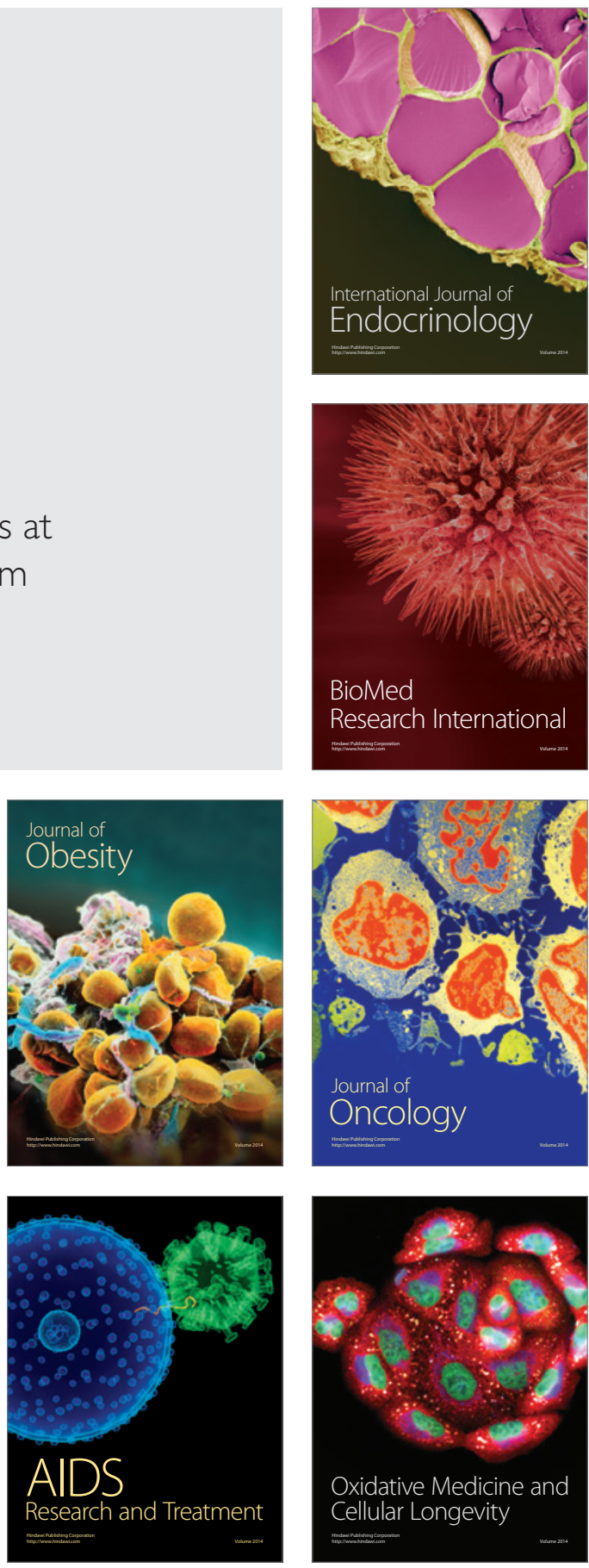